\title{
Role of miR-148a in Mitigating Hepatic Ischemia-Reperfusion Injury by Repressi the TLR4 Signaling Pathway via Targ in CaMKII $\alpha$ in Vivo and in Vitro
}

\author{
Daofeng Zheng ${ }^{\mathrm{a}}$ Zhongtang Li ${ }^{\mathrm{a}}$ Xufu Wei ${ }^{\mathrm{a}}$ Rui Liu ${ }^{\mathrm{a}}$ Ai She \\ Diao $\mathrm{He}^{c}$ Chengyong Tang ${ }^{\mathrm{d}}$ Zhongjun $\mathrm{Wu}^{\mathrm{a}}$
}

aDepartment of Hepatobiliary Surgery, The First Affiliated Hospital Chongqing, 'bepartment of Hepatobiliary surgery, Chongqing Cance 'Department of Gastrointestinal Surgery, West China Hospital, Sichual dDepartment of Clinical Pharmacology, The First Affilic 'Jospital of C. ongqing Medical University, Chongqing, China

Key Words

Ischemia-reperfusion injury MicroRNA (miRNA) • Toll-like re

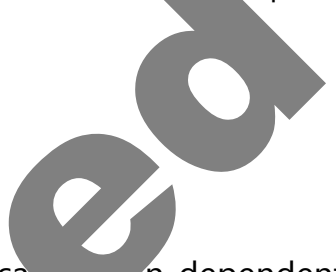

can n-dependent protein kinase II (CaMKII) • 4.(TLR4) - Hypoxia/reoxygenation

\section{Abstract}

Background/Aims: Her inflammation and un-abi cisc mia-reperfusion (I/R) injury, which is mainly induced by ellular ions, is a major negative consequence of surgery that compromises he ction. However, the exact mechanisms of liver I/R injury have not
been determin activation c the D pathway and inflammation. We previously found that miR-148a, which de ed ir...undance with increasing reperfusion time, targeted and repressed the ex $c$ on MKII . In the present study, we examined the role of the miR-148a machinery in duced. $a^{2+} /$ CaMKII and TLR4 signaling changes, inflammation, and liver dysfunction in vivo vitro. Methods: Liver function was evaluated by serum aminotransferase levels heminoxylin-eosin (HE) staining. Inflammatory factors were detected by enzyme-linked i. hosorbent assay. Gene and protein expression were assessed by RT-PCR and western slo small interfering RNA was used to silence target gene expression. HE staining and ninal deoxynucleotidyl transferase-mediated dUTP nick end labeling were used to measure hepatic tissue apoptosis. These assays were performed to identify factors upregulated in hepatic I/R injury and downregulated by miR-148a. Results: We manifested that expression of CaMKII $\alpha$ and phosphorylation of TAK1 and IRF3 were elevated in hypoxia/reoxygenation $(\mathrm{H} / \mathrm{R})$-treated primary Kupffer cells (KCs) and liver tissue of I/R-treated mice, but these effects were attenuated by treatment with miR-148a mimic and were accompanied by the

D. Zheng and Z. Li contributed equally to this article.

\begin{tabular}{ll}
\hline Zhongjun Wu & Department of Hepatobiliary Surgery, The $1^{\text {st }}$ Affiliated Hosp. of Chongqing Med. Univ. \\
& Chongqing 400016 (China) \\
& E-Mail wzjtcy@126.com
\end{tabular}


alleviation of liver dysfunction and hepatocellular apoptosis. Luciferase reporter experiments showed that miR148a suppressed luciferase activity by almost $60 \%$. Moreover, knockdown of CaMKII $\alpha$ in H/R KCs led to significant deficiencies in p-TAK1, P-IRF3, IL- 6 , and TNF- $\alpha$, which was consistent with the effects of miR-148a overexpression. Otherwise, the same trend of activation of TAK1 and IRF3 and inflammatory factors in vitro was observed in the siTAK1 + siIRF3 group compared with the siCaMKII group. Conclusion: Taken together, we conclud that miR-148a may mitigate hepatic I/R injury by ameliorating TLR4-mediated inflammation via targeting CaMKII in vitro and in vivo.

\section{Introduction}

Living donor liver transplantation is a valuable and indispen for circumventing the shortage of organs, a worldwide problem in th. transplantation. However, various problems remain unsolved, includi ro, schemia reperfusion (I/R) injury [1,2], portal hypertension [3], inflammatory, onses [-1, and graft rejection [5]. The hallmarks of hepatic I/R injury include severe cell d $\mathrm{t}$ nnd inflammatory responses that contribute to early graft failure [6]. The acti cinflan tory responses, including macrophage and neutrophil infiltration, cytokin rou and inflammatory molecular events, promotes severe insults that lead to h inflammatory responses can also be initiated and enhanced cell death [7, 8]. Despite hundreds of investigations addressing this issu he mechani. s responsible for liver I/R injury still require further exploration.

Toll-like receptors (TLRs), a group of in innate immune system receptors, have been the subject of recent research. Liv $\mathrm{K}_{\mathrm{R}} \mathrm{i}$ y can activate TLR4 through ATP generation, cellular ion (especially calcium neostasis, and generation of reactive oxygen species [9]. $\mathrm{Ca}^{2+}$ plays as a maje sec d messenger role in regulating a broad range of important cellular processes. Many ala esponses to $\mathrm{Ca}^{2+}$ signals are induced by a family of multifunctional $\mathrm{Ca}^{2+} / \mathrm{c}$ (u) ndent protein kinases (CaMKs), among which CaMKII is a ubiquitous serine/ $₹$ ine protein kinase encoded by four separate genes $(\alpha$, $\beta, \gamma$, and $\delta$ ) [10]. TLR4 ligandc can ficantly trigger elevation of intracellular $\mathrm{Ca}^{2+}$ and activation of CaMKII in $\mathrm{m} \sim$ phages. In turn, CaMKII promotes both myeloid differentiation factor 88 (MyD88)-dep ent I Toll/interleukin-1 receptor domain-containing adaptor protein inducing inter (TRIF)-dependent proinflammatory factor and type I interferon (IFN) tion oy regulating transforming growth factor- $\beta$-activated kinase 1 (TAK1) and IFN g a factor 3 (IRF3) [11]. Therefore, positive crosstalk with the $\mathrm{Ca}^{2+} /$ CaMKII pat ay i ec ired for complete activation of TLR responses in macrophages, and vice verse weve nether $\mathrm{Ca}^{2+} / \mathrm{CaMKII}$ signaling participates in inflammation of hepatic $\mathrm{I} / \mathrm{F}$ 'v into ing with TLR4 pathway has not been elucidated.

roR\ (miRNAs), a class of highly conserved small endogenous noncoding RNA mon s important post-regulators of several biological process, silence targeted NAs sinding to their 3 '-untranslated regions ( 3 '-UTRs), and are often dysregulated iv als ase and tumors $[12,13]$. In the current study, we focused on the effects of miRNAs on lammation derived from hepatic I/R injury. Ever since identification of the effects of INAs on I/R injury in myocardial ischemia and their association with angiogenesis and inhibition of apoptosis and inflammation [14], there have been more studies of the roles of miRNAs in the regulation of I/R injury and other human hepatic physiological and pathological processes [15]. One miRNA, known as miR-148a, may play an especially important role as a negative regulator of innate responses and antigen presentation in TLR-triggered dendritic cells [16]. In particular, the $3^{\prime}$-UTR of the CaMKII $\alpha$ mRNA contains the predicted target sites for miR-148a binding, which have been verified in several miRNA databases. Thus, miR-148a might ameliorate I/R by inhibiting TLR4 signaling via targeting CaMKII $\alpha$.

Although miR-148a has been widely studied with regard to its role in carcinoma and inflammation, no in vivo or in vitro studies have heretofore been published regarding the role

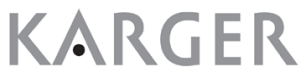




\section{Cellular Physiology Cell Physiol Biochem 2018;49:2060-2072 \\ \begin{tabular}{l|l|l} 
and Biochemistry 10.1159/000493716 & $\begin{array}{l}\text { C) } 2018 \text { The Author(s). Published by S. Karger AG, Basel } \\
\text { www.karger.com/cpb }\end{array}$ \\
\hline
\end{tabular}

of miR-148a in liver I/R injury. Previously, we had clarified that the expression of CaMKII $\alpha$ and miR-148a were negatively correlated to reperfusion time [17]. In the present study, we attempted to investigate whether miR-148a plays a vital role in alleviating liver I/R injury by suppressing TLR4-mediated inflammation. Moreover, we aimed to examine the effects of miR-148a mimics or miR-148a inhibitors on CaMKII $\alpha$ expression and TLR4-mediated inflammation in hepatic I/R injury and in an H/R treated Kupffer cells (KCs). Taken togethe our results suggest that miR-148a may alleviate hepatic I/R injury by repressing the TLR ${ }_{2}$ signaling pathway via targeting CaMKII $\alpha$ both in vivo and in vitro.

\section{Materials and Methods}

Animals

Male C57BL/6J mice (6-8 weeks old) were purchased from the Animal Center o ngqing edical University. All experimental protocols were approved by the Committee on nal $h$ at the Chongqing Medical University. Mice were maintained under a 12-h light-dark cy ith foou and water ad libitum in a temperature-controlled environment $\left(22 \pm 2^{\circ} \mathrm{C}\right.$ and $55 \pm 10 \%$ relativ nidity). The animals were maintained under laboratory conditions for an acclimatizatic or conducting the experiments.

\section{Isolation and culture of KCs}

KCs were isolated from C57BL/6J mice livers as pros sly described o . Shortly after puncturation of the portal vein, the liver was perfused with phosphate-bul. lution (Boshide, Wuhan, China) at $37^{\circ} \mathrm{C}$. The

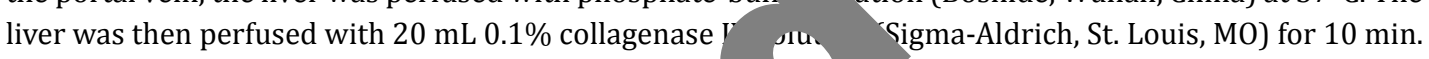
After removal of hepatic envelope, the cells were filter antr ,ed at various speeds, and washed several times. Cells were then plated in 6-well culture pl $\quad j^{\circ} \mathrm{C}$, after which the nonadherent cells were removed. The adherent KCs were cultured it alb co's modified Eagle's medium/nutrient mixture F12 medium (Gibco, Gaithersburg, MD) supplem wit $0 \%$ fetal bovine serum (Gibco), penicillin (100 U/ $\mathrm{mL})$, and streptomycin $\left(100 \mathrm{mg} / \mathrm{mI} \quad\right.$ cubated at $37^{\circ} \mathrm{C}$ in a humidified atmosphere with $5 \%$ $\mathrm{CO}_{2}$.

Animal surgery

A warm segmental $(70$ the mouse was anesthetized hepai /R model was generated as previously described [19]. Briefly, after $\%$ pentobarbital sodium and laparotomized, the arterial and portal venous blood were $f$ for ou min. Reperfusion was then performed by removing the clamp. A warming pad was provided $\mathrm{m}$ in he body temperature at $37^{\circ} \mathrm{C}$. Surgery in the sham group only exposed the portal vein fo $h$ wi $\mathrm{u}^{+}$epatic ischemia. By the end of the predetermined period following reperfusion, mice were hetiz d euthanized for tissue and plasma collection by exposure to a high concentration of $\quad \mathrm{ll} \mathrm{t}$ and sera were collected $8 \mathrm{~h}$ after reperfusion, except for detection of miR-148a mRNA abu. e at va as reperfusion times.

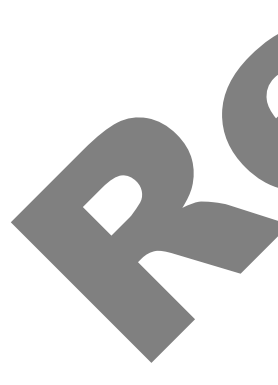

Hyp . reoxygenation model

The hypoxia/reoxygenation (H/R) model was created as previous described [20]. Hypoxia ore ditioning was performed by culturing cells in a tri-gas incubator with a $1 \%$ oxygen concentration ording to the manufacturer's instructions. The cells were then placed in a normoxic humidified incubator for various periods. All experiments were performed at $37^{\circ} \mathrm{C}$.

\section{Quantitative real-time PCR}

Total RNA was extracted using TRIzol reagent (Invitrogen, Carlsbad, CA) and reverse-transcribed in a reaction mixture. Quantitative real-time polymerase chain reaction (qRT-PCR) analysis was performed following the manufacturer's protocol. The expression data were normalized to that of glyceraldehyde 3-phosphate dehydrogenase (GAPDH). To determine miRNA expression, total RNA was reverse-transcribed, and the resulting cDNA mixed with miRNA-specific TaqMan primers (GenePharma, Shanghai, China) and TaqMan Universal PCR Master Mix (GenePharma). U6 was used as an internal 


\section{Cellular Physiology Cell Physiol Biochem 2018;49:2060-2072 \\ \begin{tabular}{ll|l} 
and Biochemistry $10.1159 / 000493716$ & $\begin{array}{l}\text { DO } 2018 \text { The Author(s). Published by S. Karger AG, Basel } \\
\text { Published online: } 22 \text { September, } 2018\end{array}$ \\
\hline
\end{tabular} \\ Zheng et al.: miR-148a Mitigates Hepatic Ischemia-Reperfusion Injury in Vivo and in Vitro}

control for normalization of miRNA levels. The primers used for these experiments are shown as follows: TAK1 forward, 5'-GCTCGCTGGTAGTGGTGTT-3', and reverse, 5'-GCCCCACTGTGAATCTGAAA-3'; IRF3 forward, 5'-TACACTGAGGACTTGCTGGAGGT- $3^{\prime}$, and reverse, $5^{\prime}$-AAGATGGTGGTCTCCTGATCC- $3^{\prime}$; CaMKII $\alpha$ forward, $5^{\prime}$-ACCTGCAC CCGATTCACAG-3', and reverse, 5'-TGGCAGCATACTCCTGACCA-3'; miR148a forward, 5'-ATGCTCAGTGCACTACAGAA-3', and reverse, 5'-GTGCAGGGTCCGAGGT-3'; GAPDH forward, 5'-AGCGAGACCCCACTAACA-3', and reverse, 5'-GGGGCTAAGCAGTTGGTG -3'; U6 forwar ${ }^{\prime \prime}$ $5^{\prime}$-CTCGCTTCGGCAGCACA-3', and reverse, 5'-AACGCTTCACGAATTTGCGT- $3^{\prime}$. The comparative threshor cycle $(\mathrm{Ct})$ method was used to measure the relative expression, where $2^{-\Delta \Delta \mathrm{t} t}$ represents the fold-change in expression, as previously described [21].

\section{Cell transfection}

KCs were plated at a density of $2-4 \times 10^{5}$ cells $/ \mathrm{mL}$ in 6-well culture plates and cultured transfected with the $50 \mathrm{nM}$ miR-148a mimic, an miR-148a inhibitor, or a negative c $r$ (Go arma) using Lipofectamine 2000 (Invitrogen) for $24 \mathrm{~h}$, referencing the manufacturer's guide KII $\alpha, \mathrm{T}, 1$, and IRF3 small interfering RNA (siRNA; GenaPharma) were transfected into KCs for 24 are shown as follows: SiCaMKIIa: 5'-CACCACCATTGAGGACGAA-3'; siTAK1: 5'-UGC AUCUU. silRF3: $5^{\prime}$-GGTTGTTCCTACATGTCTTAA-3'. H/R treatment was subsequantly pert

\section{3'-UTR luciferase reporter assays}

The pmirGLO Dual-Luciferase miRNA Target Expression Vector to perform $3^{\prime}$-UTR luciferase reporter assays. Wild-type and mutant $C$ vectors were constructed by amplifying the mouse Ca' $\alpha$ mRNA $33^{\prime}-\mathrm{L}$, , then cloning it into the XbaI site of the pmirGLO vector. KCs were co-transfected for with $80 \mathrm{ng}$ luciferase reporter plasmid, 40 ng thymidine kinase promoter-Renilla luciferase repor pio and miR-148a mimic or inhibitor (final concentration, $20 \mathrm{nM}$ ) using Lipofectamine 2000 a ding he manufacturer's instructions. Firefly luciferase and Renilla luciferase luminescence we sing the Dual-Glo Luciferase Reporter Assay System (Promega) and a GloMax 20/20 Lum om il (Promega). Data were normalized for transfection efficiency by dividing firefly luciferase activit eni luciferase activity as described previously [22].

\section{Enzyme-linked immunosorbent}

Serum levels of alanine transam (ALT) and aspartate transaminase (AST) are indices of hepatocellular injury. To deter the release of the inflammatory cytokines ALT and AST, cell supernatants and serum were collected. ease terleukin (IL)- 6 and TNF- $\alpha$ were detected using the IL-6 Mouse ELISA Kit and the Mouse TN. ISA Kit (Thermo Fisher Scientific, Waltham, MA), and release of ALT and AST were deter ing un SGPT ELISA Kit (Enzyme-linked biological technology, Shanghai, China) according to the $n$ uf 'manuals. ot

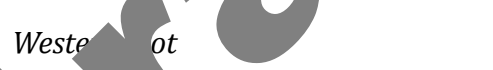

Pr teil ting was performed as the manufacturer's instructions described. Protein samples were hat from livers and KC cells by lysis buffer (Beyotime, Nantong, China). The Bradford method was $x$ to measure protein concentrations. The protein samples were separated by 5-10\% sodium cyl s. ve polyacrylamide gel electrophoresis, and gels were then transferred to a polyvinylidene fl ild membranes (Millipore, Burlington, MA). After blocking the membranes for 60 min with $5 \%$ skim sill: ley were incubated overnight at $4^{\circ} \mathrm{C}$ with anti-CaMKII $\alpha$ antibody (Cell Signaling Technology, Danvers, , anti-TAK1 antibody (Cell Signaling Technology), anti-p-TAKI antibody (Cell Signaling Technology), anti-IRF3 antibody (Cell Signaling Technology), or anti-p-IRF3 antibody (Cell Signaling Technology), and anti- $\beta$-ACTIN antibody (XinBoSheng, China). After the membranes were washed, they were probed with the corresponding secondary antibodies before developing them by enhanced chemiluminescence using a Chemiluminescent Detection Kit (Advansta, Menlo Park, CA). 


\section{Cellular Physiology \begin{tabular}{ll|l} 
and Biochemistry & $\begin{array}{l}\text { DOI: 10.1159/000493716 } \\
\text { Published online: 22 September, } 2018\end{array}$ & $\begin{array}{l}\text { (c) The Author(s). Published by S. Karger AG, Basel } \\
\text { www.karger.com/cpb }\end{array}$ \\
\hline Zhenget al. miR-148a Mitigates Hepatic Ischemia-Reperfusion Injury in Vivo and in Vitro
\end{tabular}}

Histology and terminal deoxynucleotidyl transferase-mediated dUTP nick end labeling assay

Formalin-fixed and paraffin-embedded mice liver specimens were cut into 4 micrometers sections, stained with hematoxylin and eosin (HE), and observed with light microscopy. Suzuki's criteria were used to evaluate the grade of hepatic injury [23]. Apoptosis of hepatocytes was evaluated by an In Situ Cell Death Detection Kit (Roche, Mannheim, Germany) based on terminal deoxynucleotidyl transferase-mediated dUTP nick end labeling (TUNEL). The number of TUNEL-positive cells was counted under 100× magnification + quantify the degree of apoptosis.

Ago-miR-148a therapy in mice

The C57BL/6J mice were treated by tail vein injection with ago-miR-148a (5 mg/kg; GeneP antago-miR-148a, and control ago-miRNA in saline, as previously described [24]. After $\mathrm{h}, 6$ ischemia and $6 \mathrm{~h}$ of reperfusion were performed.

Statistical analysis

Data are expressed as the mean \pm standard deviation (SD). One-way analysis $c$ Tukey-Kramer multiple comparisons tests were conducted for multiple-group c arisons, ....d Student's $t$-test was used to evaluate the significance of differences between treatments an trols. In all analyses, differences at $\mathrm{P}<0.05$ were considered statistically significant.

\section{Results}

Upregulation of CaMKII $\alpha$, $p$-TAK1, and $p$-IRF3

To assay the role of CaMKII $\alpha$, p-TAK1, and conditions, which is a well-established in vitro previous study [25], we chose $12 \mathrm{~h}$ of hypmin a. for further experiments. Western blo ${ }^{+}$ CaMKII $\alpha$, p-TAK1, and p-IRF3 protein and IRF3 protein did not incr to $\mathrm{H} / \mathrm{R}$ exhibited elevated leve consistent with the effects of hepa in vivo.

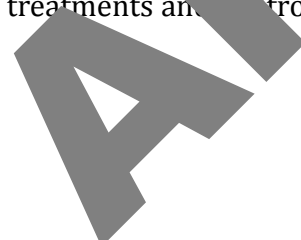
of reoxygenation as the parameters revealed that following $\mathrm{H} / \mathrm{R}$, amounts of nificantly increased, but amounts of TAK1 B). Our data suggested that KCs subjected

CaMKIIa modulate $H / R$ - luced inflammatory responses in $K C s$ via inhibiting phosphorylation of ' $\mathrm{C}$ IRF3

To determin 'fect or CaMKII $\alpha$ on the activation of TAK1 and IRF3 and H/R-related inflammation ir CS $\mathrm{A}$ ere transfected with siCaMKII $\alpha$, siTAK1, and siIRF3, respectively. Western blo ing a fo R-PCR were performed to evaluate the efficacy of transfection. The expressic CaM TAK1, and IRF3 all decreased when siRNAs were transfected into KCs (Fj 10 an Immunoassays were used to evaluate protein expression in KCs transfected to $\mathrm{ex}_{\mathrm{f}}$ vario combinations of these siRNAs. Compared with the control group, expression of 6 .a. ffec siCaMKII $\alpha$ into KCs in the H/R group, the expression of CaMKII $\alpha, p-T A K 1$, a IIRF3 revised. Interestingly, the expression of TAK1 and IRF3 remained not change. 10 jver, compared with the H/R group, the expression of TAK1, IRF3, p-TAK1, and p-IRF3 eased in the siTAK1 + siIRF3 + H/R group, while the expression of CaMKII $\alpha$ was almost the same as in the H/R group. Otherwise, the expression of p-TAK1 and p-IRF3 remained close to the same as in the siCaMKII $\alpha+H / R$ group and siTAK1 + siIRF3 + H/R groups. The observed trends in expression of the main inflammatory factors in TLR4 signaling, IL- 6 and TNF- $\alpha$, were consistent with the observed alteration in expression of p-TAK1 and p-IRF3. These results imply that CaMKII $\alpha$ may inhibit H/R-induced inflammation in KCs by targeting p-TAK1 and p-IRF3 (Fig. 1E). 


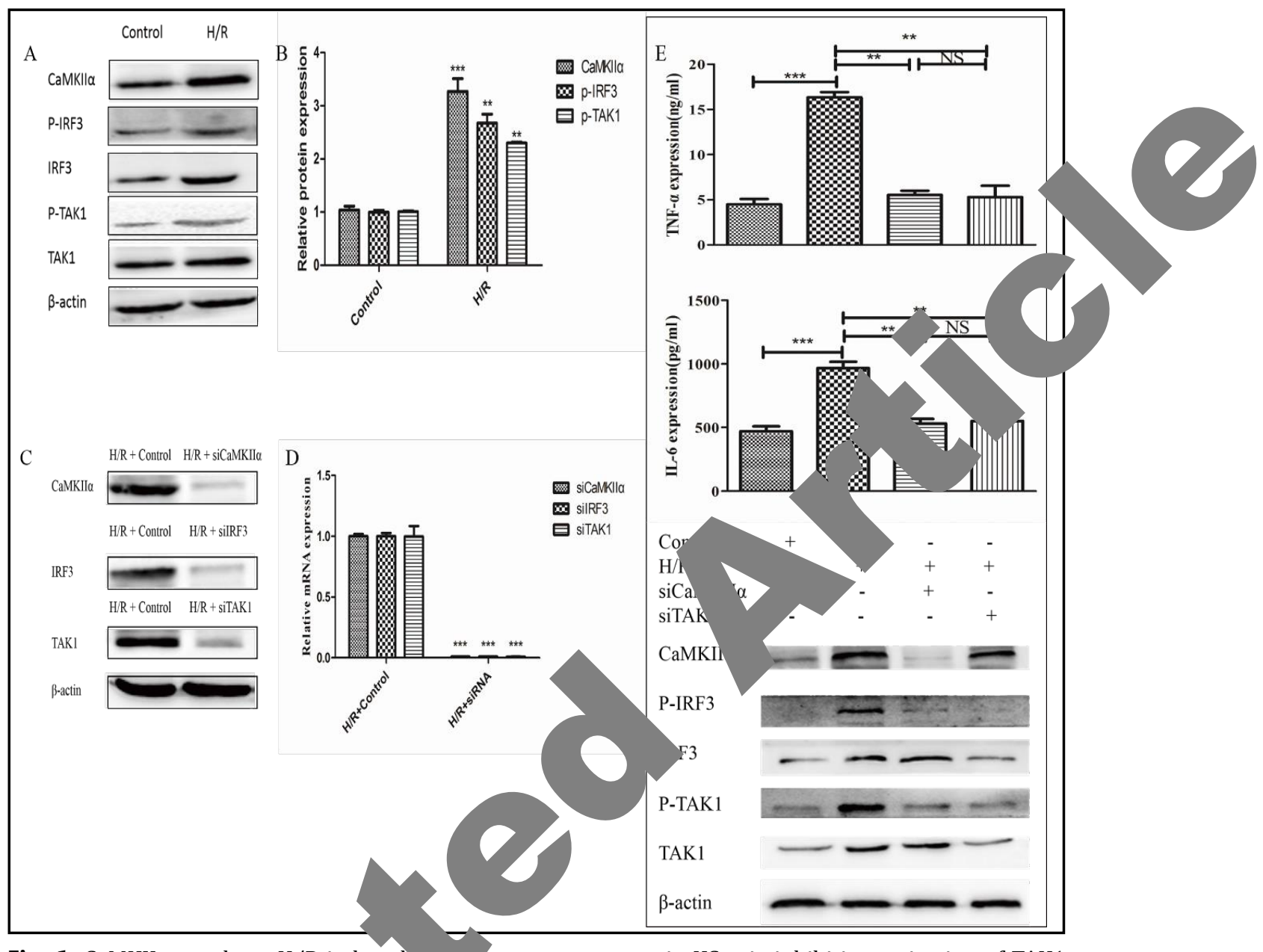

Fig. 1. CaMKII $\alpha$ regulates $\mathrm{H} / \mathrm{R}$; $\rightarrow$ ced $\mathrm{matory}$ responses in KCs via inhibiting activation of TAK1 and IRF3. A and B) qRT-PCR aysis an western blotting of CaMKII $\alpha$, TAK1, p-TAK1, IRF3, and p-IRF3 in primary KCs after H/R. C a V) $q R$ CR and western blotting were performed to evaluate the efficacy of RNA interference in - S/R- Cimary KCs after transfection with siCaMKII $\alpha$, siTAK1, or silRF3. ** $\mathrm{P}<0.01,{ }^{* * *} \mathrm{P}<0.001$ with its relative protein. E) Expression of CaMKII $\alpha, \mathrm{TAK} 1, \mathrm{p}-\mathrm{TAK} 1, \mathrm{IRF} 3$, and p-IRF3 was detec by $m$ ological assays, while the release of TNF- $\alpha$ and IL- 6 in culture supernatants was measure $y$ EL $\rightarrow$ sults are the mean \pm SD. Error bars represent SD. One-way ANOVA, t-tests, and Tukey-Kra nultip._omparisons tests were performed. NS, not significant; ${ }^{* *} \mathrm{P}<0.01 ;{ }^{* * *} \mathrm{P}<0.001$.

-148u duces H/R-mediated inflammatory responses in KCs

tigate the effect of miR-148a on inflammation in KCs, we first detected the essi of miR-148a in hepatic I/R treated mice. As shown in Fig. 2A, the expression of f $x-148$ a decreased as a function of time following reperfusion, achieving minimum levels at h $b$ elucidate the role of miR-148a on the production of proinflammatory mediators, KCs e then transfected with miR-148a mimic, inhibitor, or control, and transfection efficiency was measured by qRT-PCR. The expression of miR-148a significantly increased in the miR148a mimic-transfected group but decreased in the miR-148a inhibitor transfected group as compared with the miR-148a control group (Fig. 2B). Next, we performed ELISA assays to detect the release of inflammatory factors in supernatant. Data showed that miR-148a overexpression attenuated the production of the proinflammatory cytokines TNF- $\alpha$ and IL-6 in H/R-induced KCs, and that knocking down miR-148a aggravated this inflammation (Fig. 2C and D). Western blotting was then conducted to evaluate the protein expression. We found that the expression of CaMKII $\alpha$, p-TAK1, and p-IRF3 was elevated in the miR-148a mimic $+\mathrm{H} / \mathrm{R}$ group, but decreased in the miR-148a inhibitor $+\mathrm{H} / \mathrm{R}$ group (Fig. 2E-H).

\section{KARGER}




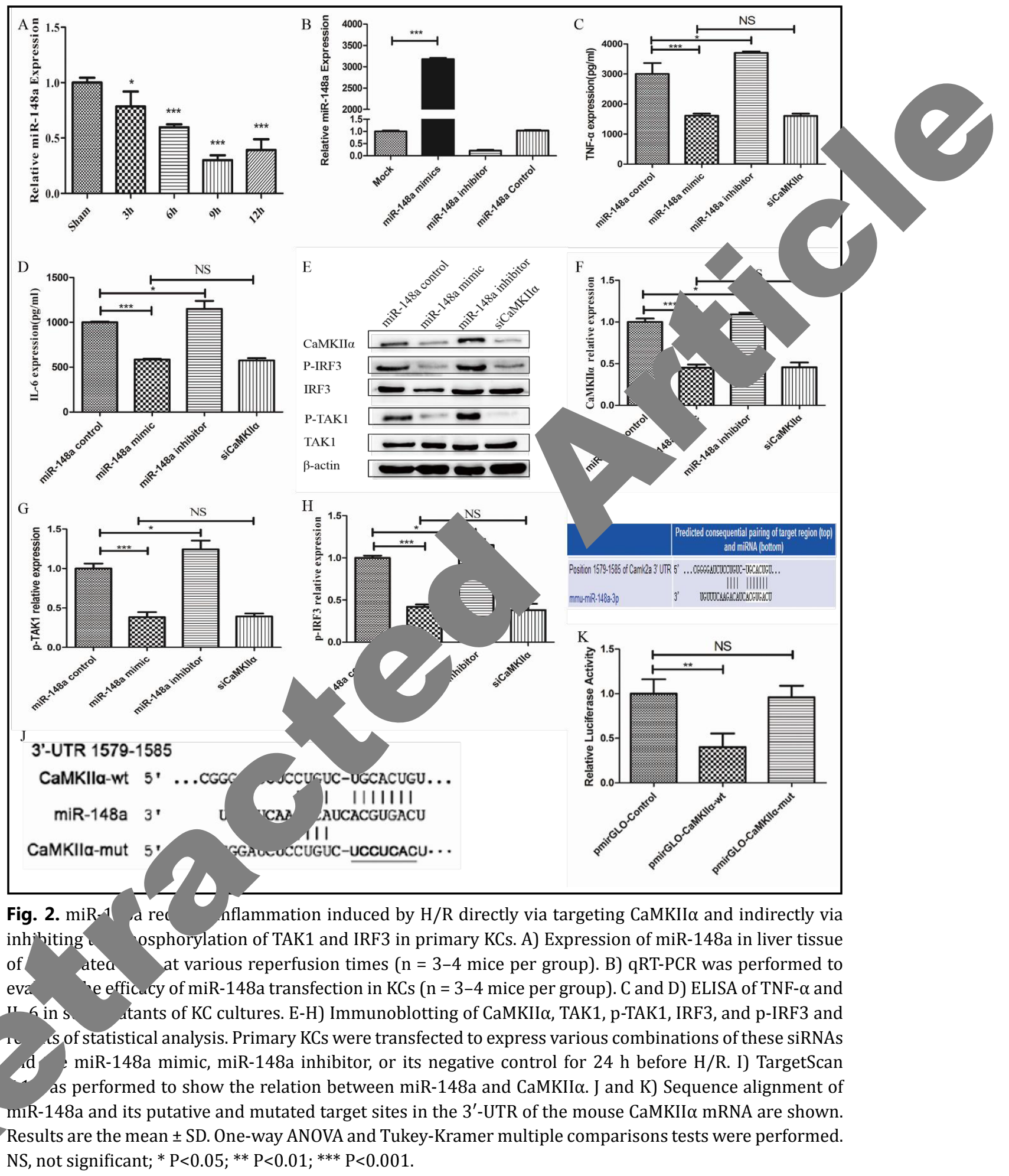


miR-148a regulates inflammation in KCs by directly targeting CaMKII $\alpha$

To evaluate whether CaMKII $\alpha$ is the main target of miR-148a influencing inflammation induced by H/R in KCs, we first used the miRNA target prediction software TargetScan 7.1 to identify putative miR-148a binding sequences in the $3^{\prime}$-UTR of CaMKII $\alpha$ (Fig. 2I). A luciferase reporter experiment was then conducted to confirm this hypothesis. Our data showed that miR148a suppressed luciferase activity by almost 60\% (Fig. 2J and K). Moreover, we als conducted siCaMKII $\alpha+\mathrm{H} / \mathrm{R}$ and miR-148a $+\mathrm{H} / \mathrm{R}$ treated KCs to elucidate whether CaMKIl is the main target of miR-148a affecting the inflammatory cascade in H/R treated KCs. There

Fig. 3. miR-148a represses hepatic i $\mathrm{n} \mathrm{fl} \mathrm{a} \mathrm{m}$ m a t o r y responses resulting from liver $\mathrm{I} / \mathrm{R}$ in C57BL/6J mice. Male mice ( $\mathrm{n}=3-4$ mice per group) were treated with ago-miR148a, antago-miR148a, ago-miRNA control, or vector for $24 \mathrm{~h}$, then subjected to I/R. A) qRT-PCR for miR-148a. B-F) qRT-PCR for CaMKII $\alpha$ and western blot analysis of CaMKII $\alpha$, TAK1, p-TAK1, IRF3, and p-IRF3. $G$ and $H$ ) ELISA of TNF- $\alpha$ and IL-6 in serum of mice. Results are the mean \pm SD. One-way ANOVA and Tukey-Kramer multiple comparise tests were perfor d. NS, not signi ant; $\mathrm{P}<0.05$; **

\section{$\mathrm{P}<0 \quad 01$}

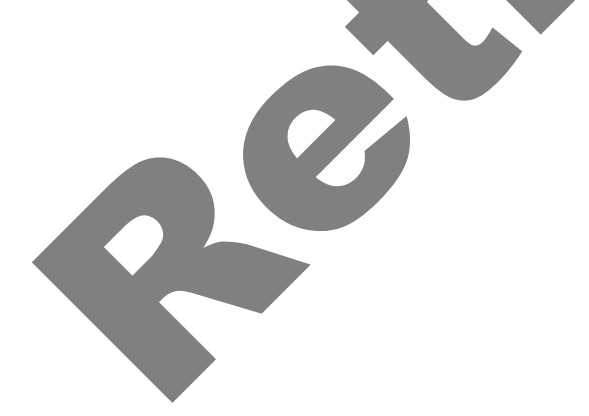

A

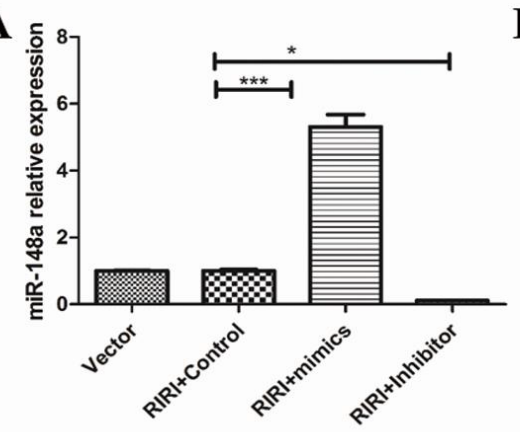

C

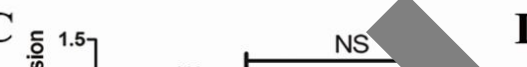

B
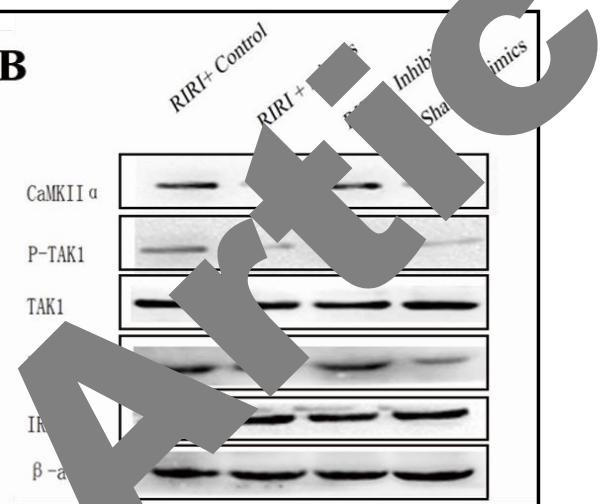

D
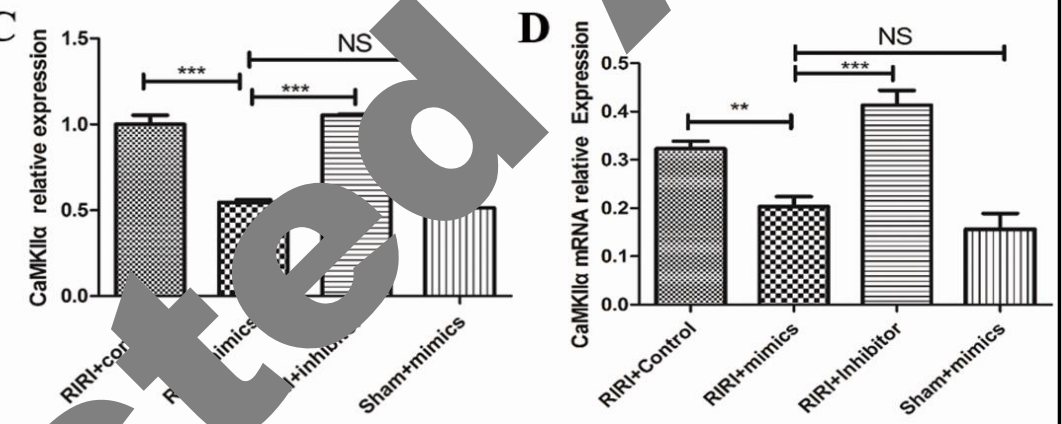

E 1.57 NS

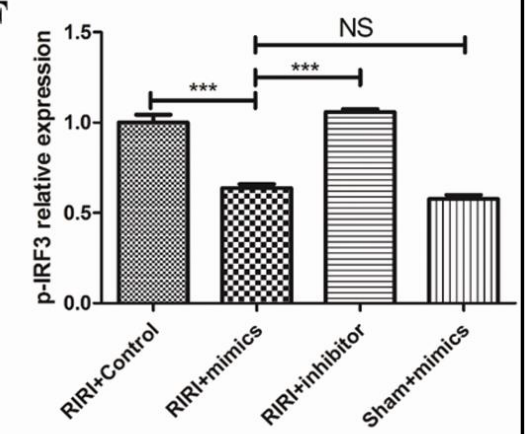

G

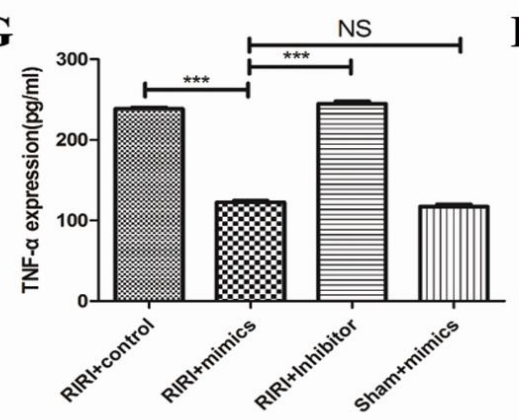

H

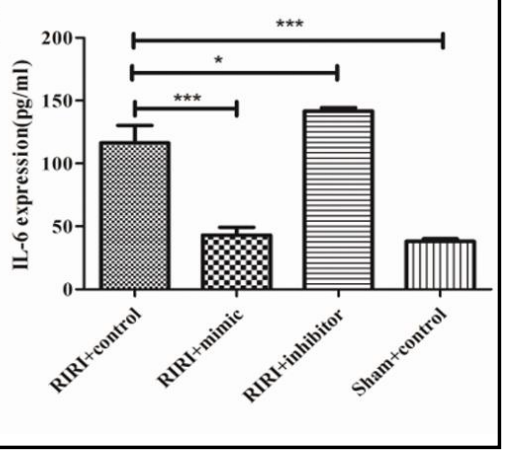




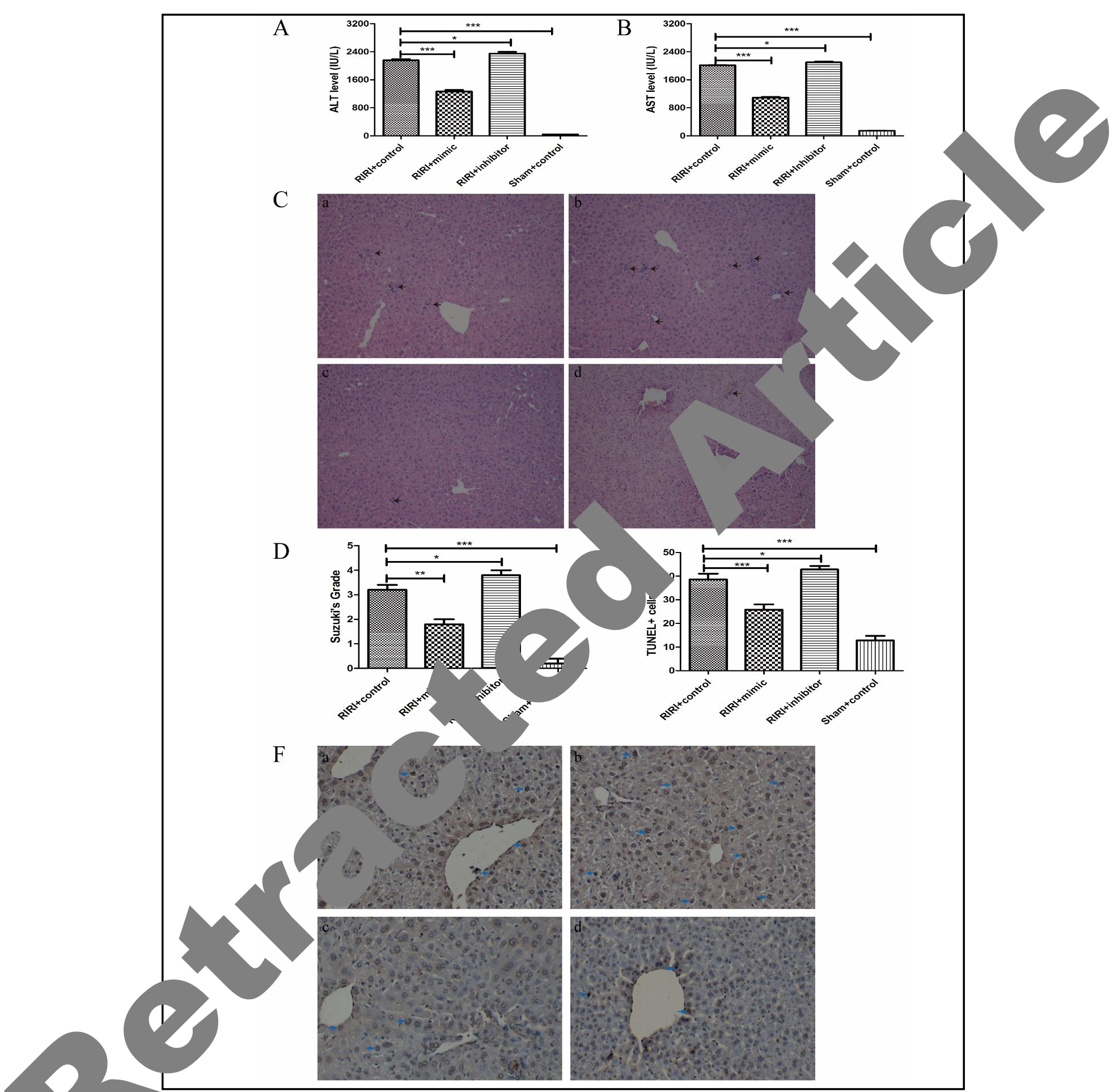

Fig. 4. Transfection of ago-miR-148a improves hepatocellular function after liver I/R injury in vivo. Male mice ( $\mathrm{n}=3-4$ mice per group) were treated with ago-miR-148a, antago-miR-148a, ago-miRNA control for $24 \mathrm{~h}$, then subjected to I/R. A and B) ELISA of ALT and AST in serum of experimental mice. C and D) Representative hematoxylin-eosin staining (100× magnification, black arrow points to the inflammatory infiltration) and Suzuki's grades. E and F) TUNEL of liver tissue (200× magnification, blue arrow points to TUNEL-positive cell), with results of statistical analysis. a, RIRI + control; b, RIRI+ inhibitor; c, RIRI + mimic; $\mathrm{d}$, Sham + control. Results are the mean \pm SD. One-way ANOVA and Tukey-Kramer multiple comparisons tests were performed. ${ }^{*} \mathrm{P}<0.05 ;{ }^{* *} \mathrm{P}<0.01 ;{ }^{* *} \mathrm{P}<0.001$.

\section{KARGER}


was no statistically significant differences between these two groups (Fig. 2C-H). These results imply that miR-148a mainly binds directly to the 3'-UTR of CaMKII $\alpha$ to decrease phlegmonosis in H/R-induced KCs.

\section{Effect of miR-148a on liver function after hepatic I/R injury.}

To verify the effect of miR-148a on hepatic I/R injury-related liver function, male mic were treated with ago-miR-148a (a chemically-modified miR-148a), antago-miR-148 a, or control ago-miRNA by tail vein injection before subjecting mice to I/R. Using qRT-PCR analysis, we measured miR-148a expression in mouse livers and found that pretreatr of mice with ago-miR-148a resulted in a significant increase in miR-148a expression preconditioning with antago-miR-148a resulted in a decrease in miR-1 compared to control ago-miRNA-treated mice (Fig. 3A). Overexpression of miR reduced CaMKII $\alpha$ expression and phosphorylation of TAK1 and IRF3 in $\mathrm{c}$ after I/R (Fig. 3B-F). Likewise, serum levels of TNF- $\alpha$ and IL-6 decreased in ago- 148a- cated mice following I/R, compared with the antago-miR-148a and control age aps (Fig. $3 \mathrm{G}$ and $\mathrm{H}$ ). To elucidate the role of miR-148a on hepatocellular functi ter I/ $h$, serum ALT and AST levels were determined in treated mice following live $\approx$ I/R. As S $\eta$ in Fig. 4A and B, compared with the control ago-miR-148a group, treatmen inhibited the release of ALT and AST induced by I/R treatm bu nent with the miR148a antagomir promoted release of ALT and AST upon I/R tre c.Further, HE and TUNEL staining were further utilized to evaluate the severity of liver injury. Compared with the ago-miR-148a control group, amelioration of $i$ mmatory ce infiltration and reduction of TUNEL-positive hepatic cells were found in $n$ verexpressed miR-148a, while these processes were more severe in the antago-miR oa up after hepatic I/R (Fig. 4C and F). Thus, our results indicate that miR-148a adm trat can protect the murine liver from $\mathrm{I} / \mathrm{R}$ injury partially via inactivation of the

\section{Discussion}

In this study, we verified that $\mathrm{m} \quad 3$ a ameliorated hepatic I/R injury and reduced liver cell apoptosis and amino coferase release by suppressing the release of proinflammatory factors through the rep sion CaMKII $\alpha$. To our knowledge, it is first time that the vital role of miR-148a in coun g hepatic I/R injury by targeting CaMKII $\alpha$ and its crosstalk with TLR4 signal of been demonstrated.

To investig $t^{\mathrm{v}}$ of miR-148a on liver I/R injury in vivo and in vitro, we transfected mice and $\mathrm{KC}$ vith to niR-148a and miR-148a mimic to overexpress miR-148a in liver and KCs. miR a ov pression significantly decreased the levels of inflammatory factors TN $\alpha \cdot n a \quad$ and apoptosis during H/R, indicating that miR-148a is an anti-inflammatory an -apop ic agent. Antago-miR-148a consistently reduced the release of TNF- $\alpha$ and IL-6 anan llular apoptosis, then attenuated hepatic function. To analyze the mechanism ese ects, we transfected KCs with miR-148a mimic, miR-148a inhibitor, or negative C tr $_{\mathrm{l}} \mathrm{l}$ miRNA into KCs. We found that transfection of miR-148a significantly suppressed a) II $\alpha, p-T A K 1$, and p-IRF3 expression, but did not influence TAK1 and IRF3 expression in KC H/R model. Furthermore, miR-148a likely interfered with the expression of CaMKII $\alpha$ through interaction with the $3^{\prime}$-UTR of CaMKII $\alpha$, which was confirmed using TargetScan 7.1 software and was consistent with the luciferase results. A previous study reported by Cao et al. also supports our findings [16]. Because miR-148a-3p can regulate more than 600 different genes, we compared the effects of siCaMKII $\alpha$ transfection with those of miR148a transfection on H/R-treated KCs. There were no statistical differences in expression of CaMKII $\alpha$, p-TAK1, and p-IRF3 proteins and inflammatory cytokines between KCs transfected with siCaMKII $\alpha$ or miR-148a, indicating that CaMKII $\alpha$ might be the main target of miR-148a for regulating inflammation in H/R treated KCs. Consistently, the overexpression of miR148a in vivo caused by ago-miR-148a transfection also decreased the levels of CaMKII $\alpha$,

\section{KARGER}


p-TAK1, and p-IRF3. Moreover, our results showed that the downregulation of CaMKII $\alpha$, p-TAK1, and p-IRF3 by miR-148a reduced the release of the proinflammatory factors TNF- $\alpha$ and IL-6. In consideration of the above-mentioned findings, we conclude that administration of miR-148a inhibits liver I/R-triggered inflammation via dephosphorylation of TAK1 and IRF3 by repressing CaMKII $\alpha$. However, whether miR-148a knockout mice are more sensitive to liver I/R injury requires further study. Moreover, recent reports indicate that miR-148which is derived from hepatocytes and released into the serum during hepatocellular injury in humans [26,27] could be chosen as a marker of liver function. Additional research should comprehensively explain the exact role of miR-148a during hepatic I/R.

Our experiments have also further elucidated the crosstalk between the CaMKI TLR4 pathways in vitro. CaMKII $\alpha$ can reduce the activity of proinflammator rote NF- $\kappa B$ and MAPK/ErK, and purinergic receptors such as TLR4 and P2RX7. In their involvement in inflammation, proteins like NF- $\kappa$ B and MAPK/ErK in pathway are known to reduce synaptic activity of CaMKII $\alpha$ by increasing it sphos ation [28-31]. Further, TAK1 and IRF3 are two key cytoplasmic protein kinas In . gnaling. Considerable research has shown that IRF3 is the key transcription $f$ ractivaud through the TRIF-dependent pathway, and that it mediates the prodyction of $t$, IFN in TLR3 and TLR4 signaling [32]. A recent report showed that downre of ph corylated TAK1 could protect against hepatic I/R injury [33]. CaMKII $\alpha$ can $\mathrm{p}$ - $\mathrm{p}_{\mathrm{l}} \quad$ these kinases and, conversely, these kinases can also upregulate the expression to fully activate the TLR4 signaling pathway and enhance the $p$ uction of proinflammatory cytokines including TNF- $\alpha$ and IL-6. We found th nockdown 0 AK1 and IRF3 significantly reduced the protein expression of TAK1, IRF3 an phosphorylated form p-IRF3, and the release of inflammatory mediators in $\mathrm{KCs}$ fo $\mathrm{Y}^{\prime} \mathrm{Im}_{\mathrm{R}}$ in vitro. Moreover, transfection of KCs with siCaMKII $\alpha$ decreased the express of AK1 and p-IRF3 and the secretion of TNF- $\alpha$ and IL-6, but did not affect th ${ }^{2}$ TAK1 or IRF3. Finally, there was no statistical difference in the expression o $-1,1$ and p-IRF3 or in the release of inflammatory cytokines in the siCaMKII $\alpha+$ H/R gro $m$ red with the siTAK1 + silRF3 + H/R group. These data suggest that $\mathrm{p}$-TAK $\mathrm{p}$ - e important targets of CaMKII $\alpha$ for reducing proinflammatory cytokine relc $\mathrm{H} / \mathrm{R}$ treated KCs. Our findings were consistent with previous research on RAW 44.7 with LPS by Liu et al. [11]. The TLR4 signaling pathway activates differe rrabepatic cell types, including dendritic cells [34], sinusoidal endothelial cells [35], a hepc cytes [36], and the effects of miR-148a and CaMKII $\alpha$ on these other cell typecrec, ther investigation.

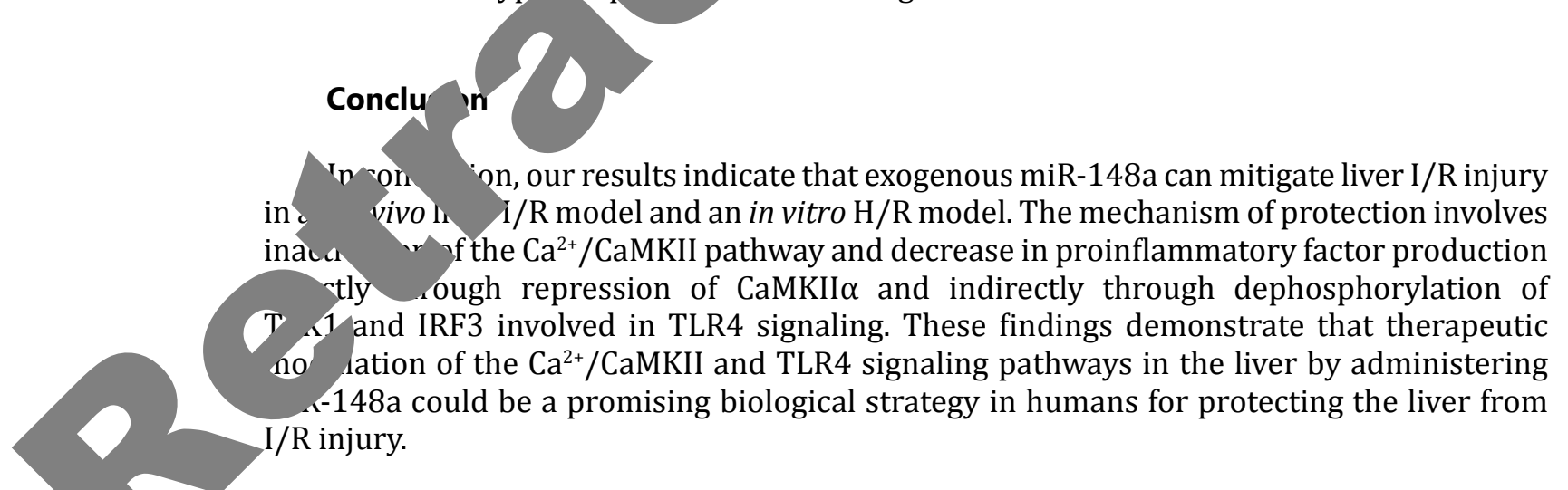

\section{Acknowledgements}

We thank the members of our laboratory for their technical comments and helpful discussions. This study was supported by grants from the National Natural Science Foundation of China (No. 81672959) and the Natural Science Foundation of Chongqing, China (No. cstc2015shmszx120019). 


\section{Cellular Physiology Cell Physiol Biochem 2018;49:2060-2072 and Biochemistry DOI: 10.1159/000493716 2018 (O) 2018 The Author(s). Published by S. Karger AG, Basel

\section{Disclosure Statement}

article.

The authors declare that they have no conflicts of interest regarding the contents of this

\section{References}

1 Theodoraki K, Arkadopoulos N, Nastos C, Vassiliou I, Karmaniolou I, Smyrniotis V: Small liver remnan more vulnerable to ischemia/reperfusion injury after extended hepatectomies: a case-control stud J Surg 2012;36:2895-2900.

2 Wu J, Tang Q, Shen J, Yao A, Wang F, Pu L, Yu Y, Li X, Li G, Zhang F, Sun B, Kong L, Li D, Zhang Y, $\mathrm{X}$ : Comparative proteome profile during the early period of small-for-size liver trans revealed the protective role of Prdx5. J Hepatol 2010;53:73-83.

-3 Onoe T, Tanaka Y, Ide K, Ishiyama K, Oshita A, Kobayashi T, Amano H, Tashiro H, O Portal Hypertension by Continuous Portal Infusion of PGE1 and Immunologic Living-Donor Liver Transplantation. Transplantation 2013;95:1521-" -27.

-4 Yang ZF, Ho DW, Chu AC, Wang YQ, Fan ST: Linking inflammation to allografts: the potential role of early macrophage activation. Am J Ti Liang TB, Man K, Lee TKW, Tsui SHT, Lo CM, Xu X, Zheng SS, Fan ST, W

\section{lar} tion in all-for-size liver 196-209.

stinct intragraft response 3;75:673-678. pattern in relation to graft size in liver transplantation. Transplantatio Zhai Y, Busuttil RW, Kupiec-Weglinski JW: Liver ische nd reperfusio njury: new insights into mechanisms of innate-adaptive immune-mediated tissu $\quad$ mmation. Am J Transplant 2011;11:15631569. Zhai Y, Petrowsky H, Hong JC, Busuttil RW, Kupiec- $И$ transplantation from bench to bedside. NatP

-8 Vardanian AJ, Busuttil RW, Kupiec-Weglin JW. olf fular mediators of liver ischemia and reperfusion injury: a brief review. Mol Med 2 $08 ; 14: 3$

10 BraunAP, Schulman H: Tr aurtifur tional calcium/ calmodulin-dependent protein kinase: from form to function. Annu Rev Phy: 1995; 417-445.

11 Liu X, Yao M, Li N, Cao X: CaMKII promotes TLR-triggered proinflammatory cytokine and type I inte duction by directly binding and activating TAK1 and IRF3 in macrophages. Blood 2008;112:49 $4 \mathrm{C}$ 0.

12 Klune JR ung cular biology of liver ischemia/reperfusion injury: established mechanisms and recer ncemu.... Surg Clin North Am 2010;90:665-677.

$>13$ Y Y hanism, innate-adaptive immune-mediated tissue inflammation. Am J Transplant 2011;11:15631.

Eltzšng HK, Eckle T: Ischemia and reperfusion from mechanism to translation. Nat Med 2011;17:139101.

5 Jang XW, Heegaard NH, Orum H: MicroRNAs in Liver Disease. Gastroenterology 2012;142:1431-1443. Liu X, Zhan Z, Xu L, Ma F, Li D, Guo Z, Li N, Cao X: MicroRNA-148/152 impair innate response and antigen presentation of TLR-triggered dendritic cells by targeting CaMKII $\alpha$. J Immunol 2010;185:7244-7251. Zheng D, He D, Lu X, Sun C, Luo Q Wu Z: The miR-148a alleviates hepatic ischemia/reperfusion injury in mice via targeting CaMKII $\alpha$. Xi Bao Yu Fen Zi Mian Yi Xue Za Zhi 2016;32:1202-1206.

18 Pei-zhi Li, Jin-zheng Li, Min Li, Jian-ping Gong, Kun He: An efficient method to isolate and culture mouse Kupffer cells. Immunol Lett 2014;158:52-56.

19 Shen XD, Ke B, Zhai Y, Amersi F, Gao F, Anselmo DM, Busuttil RW, Kupiec-Weglinski JW: CD154-CD40 T-cell costimulation pathway is required in the mechanism of hepatic ischemia/reperfusion injury, and its blockade facilitates and depends on hemeoxygenase- 1 mediated cytoprotection. Transplantation 2002;74:315. 


\section{Cellular Physiology Cell Physiol Biochem 2018;49:2060-2072

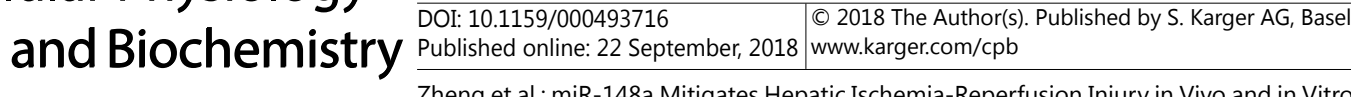 \\ Zheng et al.: miR-148a Mitigates Hepatic Ischemia-Reperfusion Injury in Vivo and in Vitro}

20 Jiang W, Kong L, Ni Q Lu Y, Ding W, Liu G, Pu L, Tang W, Kong L: miR-146a Ameliorates Liver Ischemia/ Reperfusion Injury by Suppressing IRAK1 and TRAF6. PLoS One 2014;9:e101530.

-21 Livak KJ, Schmittgen TD: Analysis of Relative Gene Expression Data Using Real-Time Quantitative PCR and the 2- $\Delta \Delta$ Ct Method. Methods 2001;25:402-408.

-22 Wei X, Tang C, Lu X, Liu R, Zhou M, He D, Zheng D, Sun C, Wu Z: MiR-101 targets DUSP1 to regulate the TGF- $\beta$ secretion in sorafenib inhibits macrophage-induced growth of hepatocarcinoma. Oncotarget 2015;6:18389-18405.

-23 Suzuki S, Toledo-Pereyra LH, Rodriguez FJ, Cejalvo D, Ke B: Neutrophil infiltration as an important factor in liver ischemia and reperfusion injury. Modulating effects of FK506 and cyclosporine. Transplantatio 1993;55:1265-1272.

24 Wang X, Guo B, Li Q, Peng J, Yang Z, Wang A, Li D, Hou Z, Lv K, Kan G, Cao H, Wu H, Song J, S, Li Y, Zhu M, Zhang P, Peng S et al.: miR-214 targets ATF4 to inhibit bone formation. Nat M 100.

25 He D, Guo Z, Pu JL, Zheng DF, Wei XF, Liu R, Tang CY, Wu ZJ: Resveratrol preconditioni hepatocytes against hepatic ischemia reperfusion injury via Toll-like receptor $4 / \mathrm{n}$ pathway in vitro and in vivo. Int Immunopharmacol 2016;35:201-209.

-26 Lucile Amrouche, Marion Rabant, Dany Anglicheau: MicroRNAs as bicmarkers o. Rev (Orlando) 2014;28:111-118.

27 Farid WR, Pan Q, van der Meer AJ, de Ruiter PE, Ramakrishnaiah V, HL, Metselaar HJ, Tilanus HW, Kazemier G, van der Laan LJ: Hepatocy Biomarkers of Hepatic Injury and Rejection after Liver Transplantation $\quad$ er Transp 2012;18:290-297.

28 Meffert MK, Baltimore D: Physiological functions for NF-kappaB. A d As Neurosci 2005;28:37-43.

29 Luo F, Yang C, Chen Y, Shukla P, Tang L, Wang LX, Wang versal of chronic inflammatory pain by acute inhibition of $\mathrm{Ca} 2+/$ calmodulin-dependent protein k

-30 Gómez-Villafuertes R, García-Huerta P, Díaz-Hernán I, M ) ortugal MT: PI3K/Akt signaling pathway

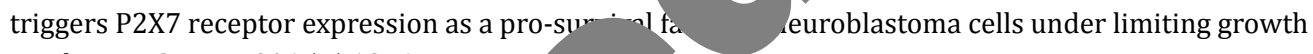
conditions. Sci Rep 2015;5:18417.

31 Huang W, Ghisletti S, Perissi V, Rosenfeld signaling at the NCoR derepress

-32 Honda K, Taniguchi T: IRFs: mas ${ }^{+}$ recognition receptors. Nat Rev Immu.

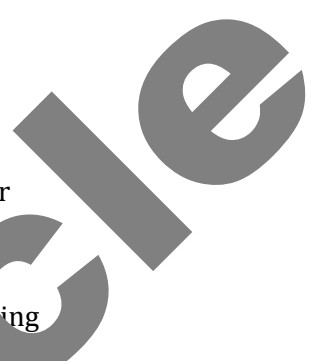
; ;:644-658.

-33 Hu J, Zhu XH, Zhang XJ, W X, Zhang K, Zhang P, Zhao GN, Gao L, Zhang XF, Tian S, Li H: Targeting TRAF3 signaling protects again epatic hemia/reperfusions injury. J Hepatol 2016;64:146-159.

-34 De Creus A, Abe M, Lau A An H, Raimondi G, Thomson AW: Low TLR4 expression by liver dendritic cells correlates uceu capacity to activate allogeneic $\mathrm{T}$ cells in response to endotoxin. J Immunol 2005;174:20 20

-35 Uhrig A, ' harsc R, ${ }^{\prime}$ emer M, Hegenbarth S, Hamann A, Neurath M, Gerken G, Limmer A, Knolle PA: Devel ant an tional consequences of LPS tolerance in sinusoidal endothelial cells of the liver. J Le koo 2005;77:626-633.

36 sumu co A, Takii T, Hayashi H, Onozaki K: Endotoxin and cytokine regulation of toll-like receptor TLR4 gene expression in murine liver and hepatocytes. J Interferon Cytokine Res 2000;20:915-

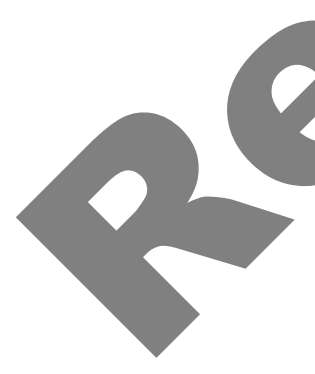

